\title{
HIV encephalopathy presenting as schizophrenia-like delusions during highly active antiretroviral therapy (HAART) case report
}

\author{
Mikako Fuji $^{1}$, Misaki Iseki ${ }^{2}$, Seisho Takeuchi ${ }^{3}$, Kyoko Kakeda $^{1}$, Shinji Shimodera $^{{ }^{*}}$, \\ Hiromi Seo $^{3}$, Shimpei Inoue ${ }^{1}$
}

\footnotetext{
${ }^{1}$ Department of Neuropsychiatry, Kochi Medical School, Kochi University, Kochi, Japan;

${ }^{2}$ Department of Psychiatry, Ichiyou Hospital, Kochi, Japan;

${ }^{3}$ Department of Medicine, Kochi Medical School, Kochi University, Kochi, Japan.

Email: shimodes@kochi-u.ac.jp
}

Received 6 August 2011; revised 11 September 2011; accepted 25 September 2011.

\begin{abstract}
After HAART (highly active antiretroviral therapy), which is a powerful antiviral drug regimen, was introduced into HIV therapy for AIDS patients, AIDS mortality decreased dramatically. The incidence of encephalopathy among AIDS patients was reported to be $10 \%$ to $40 \%$ in the past, but the introduction of HAART resulted in a decrease in HIV encephalopathy and an increase in mild CNS-related deficits, which affect the quality of life of AIDS patients. We report the case of an AIDS patient who developed schizophrenia-like delusions associated with encephalopathy. Administration of an antipsychotic drug and hypnotics was effective in relieving the symptoms of patients with insomnia, depressed mood, and delusions. Further information on the neuronal mechanism underlying the mental symptoms observed in our patient will be necessary to understand the process of pathogenesis of the encephalopathy and to devise an adequate clinical strategy for treatment.
\end{abstract}

Keywords: HIV; Encephalopathy; Schizophrenia; HAART; Antipsychotics

\section{INTRODUCTION}

There have still been few reports regarding HIV encephalopathy in Japan. 1) According to a WHO report, since the 1990s the mean worldwide HIV infection rate of the adult population (15 to 45 years of age) has been in the $0.8 \%$ range. 2) Ever since the powerful antiviral therapy consisting of combinations of several anti-HIV drugs, called HAART (highly active antiretroviral therapy), became established, the numbers of persons who have developed AIDS and died has been decreasing. On the other hand, as a result of the advent of HAART, the life span of HIV patients has grown longer, and the number of patients who develop HIV encephalopathy has increased 3, 4, 5) HIV encephalopathy often develops when the CD4 cell count drops below100/ $\mu$, and before HAART therapy was established, it was observed in $10 \%-40 \%$ of the patients. Now that HAART has been established, the CD4 cell count of many HIV patients is above $200 / \mu \mathrm{l}$, and HIV encephalopathy has decreased. 6) We report the case of a patient who, despite good control of his HIV infection, progressed to HIV encephalopathy and exhibited a schizophrenia-like hallucinatory delusional state that was associated with it.

\section{CASE REPORT}

[Patient] A 38-year-old male whose premorbid personality was introverted and stubborn. His elementary, junior high, and high school grades were average.

[Chief complaint] A hallucinatory delusional state. [Complications] Hypertension.

[Family history] Mother: Schizophrenia, hypertension. Died of a ruptured cerebral artery aneurysm. Father: Hypertension. Died of a cerebral hemorrhage. Nephew: Epilepsy, mental retardation.

[Drinking history] None.

[Smoking history] None.

[History of use of psychoactive substances, such as narcotics] None.

[History of the present illness] The second of two children. Growth and development unremarkable. Both parents died while the patient was in high school, and his sister, who was 7 years older, became a parental substitute. After graduating from a vocational-technical school, he found employment at age 20, but after working for a company for 2 years, he was unable to stand the pressure 
of the work, and resigned. He subsequently performed a series of part-time jobs. The patient is homosexual.

At age 26, he was diagnosed with HIV infection. In July of the same year, while an inpatient in the internal medicine department of university hospital A in another prefecture for Pneumocystis pneumonia, he attempted suicide because of anxiety about dying. At age 28 , he was admitted for cytomegalovirus retinitis in September, but his visual impairment progressed, and he developed insomnia and suicidal ideation. The patient was examined in the psychiatry department of the hospital because he had taken an overdose of a hypnotic agent, and he was diagnosed with reactive depression. His symptoms improved in response to treatment with etizolam $1.5 \mathrm{mg}$ and mianserin $20 \mathrm{mg}$. Antiviral drug therapy was subsequently instituted in the internal medicine department of the same hospital (zidovudine monotherapy and in combination with didanosine, lamivudine, nelfinavir, etc). At age 29 , treatment with a combination of zidovudine 200 $\mathrm{mg}$, lamivudine $300 \mathrm{mg}$, nelfinavir $2500 \mathrm{mg}$ was started in May. He had stopped working because of the visual impairment, was receiving public assistance, and continued to live alone. At the time his HIV-RNA load was under 50 copies per $\mathrm{ml}$, his CD4 cell count was $700 / \mu \mathrm{l}$ $1000 / \mu$, his HIV infection was well controlled, and his cognitive functions and personality had been maintained.

At 34 years of age, in mid-October the patient began to hear the voice of a neighbor saying negative things about him, and he started to avoid his home and spend all day outdoors. In early November, when he was walking around completely nude and banging his head against walls, he was taken into police custody and admitted to the internal medicine department of the same hospital. He was treated with haloperidol $5 \mathrm{mg}$ i.v., but because the delusions and hallucinations persisted, two weeks later he was transferred to the psychiatry department of the same hospital. Hallucinations and delusions, a memory disorder, and a consciousness disorder were noted, and because at a time when his mental symptoms became more severe an unenhanced $\mathrm{T} 2$ high-signal lesion was observed in the head of the right caudate nucleus on an MRI examination of the head, reduced blood flow in the occipital lobe and anterior cingulate gyrus bilaterally on a SPECT examination, and waves predominantly in the occipital lobe and sharp waves in the right occipital area on an EEG, a diagnosis of HIV encephalopathy was made. His scores on the WAIS-R at the time were: VIQ 78, PIQ 46, full-scale IQ 61. The mental symptoms were improved by treatment with haloperidol $12 \mathrm{mg}$ and chlorpromazine $25 \mathrm{mg}$, and he was discharged in February of the following year. The WAIS-R administered before he was discharged showed an improvement to: VIQ 84, PIQ 63, and full-scale IQ
73. There were no changes in his HIV drugs or their doses during the course of his illness. Perospirone $16 \mathrm{mg}$ was substituted because of extrapyramidal symptoms. The patient later moved close to his sister and lived alone in an apartment with the assistance of a helper 3 times a week. In July of the same year the patient was referred to our department by a local physician and examined. He was transferred to the internal medicine department of our hospital for treatment of the HIV infection, and HAART was continued with no changes to the regimen prescribed. Management in our department consisted of substituting quetiapine $50 \mathrm{mg}$ because mild extrapyramidal symptoms had been noted, but the dose was reduced to $25 \mathrm{mg}$ in November, because the mental symptoms had stabilized, and quetiapine was discontinued in January of next year. Thereafter only zolpidem 10 $\mathrm{mg}$ and etizolam were prescribed for insomnia, and the mental symptoms were stable. The patient's HIV-RNA load was under $50-74$ copies $/ \mathrm{ml}$, and the CD4 cell count was $700 / \mu \mathrm{l}-1000 / \mu \mathrm{l}$, and thus his HIV infection was also well controlled.

At age 37, a feeling of residual urine developed around November, and the patient became extremely anxious, thinking that it was a symptom caused by progression of the HIV infection. Because he developed a depressed mood, decreased motivation, a reduced appetite, and insomnia in mid-December, he was started on sertraline $25 \mathrm{mg}$. Living alone later became difficult, and the patient moved in with his sister. At age 38, auditory hallucinations developed in early January. Because such hallucinations and delusions as "A police officer came in a patrol car, and I talked to the police officer" developed in addition to the depression, the dose of sertraline was increased to $50 \mathrm{mg}$, and risperidone $2 \mathrm{mg}$, levomepromazine $25 \mathrm{mg}$, and diazepam $10 \mathrm{mg}$ were added. When the patient subsequently developed dysuria in addition to poor oral intake, he was admitted to our department as an emergency 7 days after the onset of the hallucinations and delusions.

[Mental symptoms on admission] The patient tended to be drowsy. He was able to answer questions by shaking his head, but he spoke very little. There was no disorientation. A delusion that "I will be picked up by the police" and hallucinations whose content was unknown were noted. Oral intake was poor. The patient resisted being examined, and he finally closed his eyes and no longer responded to questions. The findings resembled the catatonic manifestations of schizophrenia.

[Physical findings] Body temperature: $37.4 \mathrm{C}$, blood pressure: 199/147 mmHg, pulse: 116/min, SpO2: 97\% (room air).

[Neurological findings] No nuchal rigidity. Kernig sign present. No limb paralysis. No sensory abnormaliti- 
es. Babinski reflex and Chaddock reflex positive.

[Test findings on admission] MRI of the head: A wide high intensity area was seen in the brain stem, around the anterior horn of the lateral ventricles, and in the subcortical white matter on T2WI.

Cerebrospinal fluid examination: Cell count 27/3 $\mu$, protein $84 \mathrm{mg} / \mathrm{dl}$, Cryptococcus neoformans (-), tubercle bacillus (PCR method) (-), cytomegalovirus antigen (-), JC virus antigen (-), HIV-RNA $9.4 \times 100$ copies.

EEG findings: A low wave occurrence rate and $\alpha$ wave burst suppression pattern were noted.

[Test findings during the subsequent course] EEG findings on hospital day $10: 8-10 \mathrm{~Hz}, 30$ - $50 \mathrm{~V}$ occipital lobe predominant waves were noted.

Blood examination: HIV-RNA load 31000 copies/ml, CD4 cell count 397/ $\mu$.

MMSE: 16/22 points (conducted omitting the 3-step command, reading, naming, and writing and constructional praxis, which require visual acuity. Unable to answer two questions, i.e., region and floor of the building, in regard to orientation to place, and one of three questions in regard to recall.) WAIS-R: VIQ 82 (because of the visual impairment, the PIQ test with blocks, symbols, etc., was not performed. The VIQ test alone was performed). HAM-D on hospital day 18:19 points

[Hospital course] A $1500 \mathrm{ml} /$ day i.v. infusion was administered to treat dehydration. Based on his course the patient was concluded to have been overly sedated by the psychotropic drugs, and the oral psychotropic medication was discontinued. The anti-HIV drugs were stopped because of his poor oral intake. An EEG was performed on hospital day 2, the findings described above were observed, and a mild consciousness disorder was suspected. Oral intake became possible on hospital day 5 . Communication had improved in comparison with on admission, but his reactions to conversation were slow, and an attention deficit disorder was observed. The patient was capable of simple communication in everyday life, but he could not converse about difficult content.

Schizophrenia-like symptoms were observed, such as bizarre speech and conduct in which he seemed to be having delusions, e.g., "Stealing time...," or behavior in which he appeared to be listening to an auditory hallucination and suddenly uttered a word as though he were conversing with the auditory hallucination, etc. In addition, there was self-accusatory speech, saying "I'm receiving public assistance, and I'm taking life easy every day," and a tendency to stay in bed and loss of motivation were observed. An EEG was performed again on hospital day 10, and signs of an improvement in the consciousness disorder, such as an increase in the rate of occurrence of waves, were noted. On the same day, quetiapine $25 \mathrm{mg}$ was started to treat his mental symp- toms, and all of the hallucinations and delusions subsequently resolved. While nursing care was necessary because of the patient's impaired vision, he had become familiar with the hospital unit and was largely capable of independent living. On hospital day 14 he was examined in the neurology department, and HIV encephalopathy was diagnosed based on a cerebrospinal fluid examination and the imaging findings. No improvement in the dysuria was observed even after the psychotropic drugs were discontinued. Based on the diagnostic images of the brain the dysuria was judged to be a disturbance caused by the HIV encephalopathy lesions, and it was decided to continue the urinary catheterization. Symptoms of depression, such a decreased motivation, diminished interest, etc., were noted, but on hospital day 15 the patient's score on the HAM-D was 19 points, and his course was monitored without prescribing any antidepressant medication. There was also a slight improvement in the patient's response to conversation and his interest in the other person, but he did not recover to his condition prior to the present episode. On hospital day 42 he was discharged to his sister's home.

[Post-discharge course] The patient continued to live with his sister after being discharged from the hospital. He gradually regained his vitality, and his response to conversation also returned to its previous level. His mental symptoms stabilized at quetiapine $25 \mathrm{mg}$. Because his HIV-RNA load on post-discharge day 36 was high, 130,000 copies/ml, and his CD4 cell count had declined even further to $299 / \mu 1$, the patient was concerned about a progression of his symptoms, and he wanted to resume HAART. It was concluded that he was capable of taking his medication regularly, and on post-discharge day 54 HAART was restarted. On post-discharge day 82 his HIV-RNA load had decreased to 680 copies/ml, and his CD4 cell count had risen to $678 / \mu 1$.

\section{DISCUSSION}

It is generally said that HIV encephalopathy often develops when the CD4 cell count is below 100/1.6) Even though our patient's HIV infection was well controlled by HAART, with an HIV-RNA load under 50 copies /ml and a CD4 cell count of $700-1000 / \mu 1$, he developed HIV encephalopathy and exhibited a hallucinatory delusional state, and the symptoms improved in response to psychotropic drugs without changing the content of the HAART regimen. The hallucinations and delusions did not recur after that, even when the psychotropic medication was stopped, and his HIV infection was well controlled, with an HIV-RNA load of under 50 to 74 copies $/ \mathrm{ml}$ and a CD4 cell count of $700-1000 / \mu 1$, however, a relapse of the HIV encephalopathy was observed 4 years later, and he once again exhibited signs of hallucinations 
and delusions. Although our patient had a past history of Pneumocystis pneumonia and cytomegalovirus retinitis, he had no clear history of central nervous system infection other than the retinitis. Since both a process of improvement of the consciousness disorder and resolution of the mental symptoms were observed in regard to the patient's hallucinations and delusions, the course of treatment appeared to be different from that of schizophrenia. In the future it seems that it will be necessary to bear in mind HIV encephalopathy cases such as our own, in which the patient exhibited mental symptoms, including hallucinations and delusions, as a result of a longterm central nervous system disturbance, and examine the patients even when their CD4 cell count is adequate. At age 28 , our patient's CD4 cell count was below $10 / \mu 1$ in September, and he developed cytomegalovirus retinitis as a complication. It was estimated that he had probably contracted the HIV infection more than 20 years previously. After infection in the periphery, HIV rapidly migrates centrally, and it causes behavior disorders, motor disorders, and cognitive dysfunction. Our patient's mother had schizophrenia, but it is unclear whether that contributed to his vulnerability to hallucinations and delusions.

The powerful antiviral therapy call HAART was introduced in Japan about 10 years ago, and the number of patients who die of HIV infection declined, but at that time there was a very harsh situation in which life and death were governed by whether HAART had been introduced in the AIDS unit. Our patient had been admitted to the internal medicine department of hospital A for Pneumocystis pneumonia 3 years before, but at that time HAART had not been established, and presumably the patient became depressed and planned to commit suicide as a result of anxiety of about dying because of seeing patients around him die day after day and thinking that one day the same would probably happen to him. The patient developed cytomegalovirus retinitis as a complication at precisely that time, and he was one of the first patients to receive the benefits of HAART after it became available in Japan. Some of the drugs used in HAART easily pass through the blood-brain barrier, and others do not. Zidovudine has the highest transfer rate of the drugs used to treat our patient, i.e., zidovudine, lamivudine, and nelfinavir, and it has been reported to be effective against HIV encephalopathy. 7) The fact that only a $200 \mathrm{mg}$ dose of zidovudine, i.e., one third the usual dose, could be used in our patient because of impaired hematopoiesis may have played a role in the development of the HIV encephalopathy.

Before HAART was established, HIV encephalopathy was observed in $10 \%-40 \%$ of AIDS patients, but at present the proportion has declined to $10.5 \%$. On the other hand, the life span of HIV patients has increased as a result of the advent of HAART, and the number of pa- tients like our own who develop HIV encephalopathy despite receiving HAART has risen, 4) Symptoms of HIV encephalopathy, such as cognitive dysfunction, memory disorders, etc., greatly reduce the QOL (quality of life) of patients with HIV infection. When, as in our own patient, a long time has passed after being infected, efforts at early detection and prevention of progression of HIV encephalopathy by means of the HIV Dementia Scale and even more precise psychological tests, 8) and quantitative determinations of viral load in cerebrospinal fluid and other substances that serve as markers, 9) seem necessary, even in cases that have been well controlled by HAART.

\section{CONCLUSIONS}

HIV testing of schizophrenia patients is rare in Japan. As in our own patient, if there is no advance information in relation to HIV, there is a strong possibility of HIV encephalopathy not being detected or taking too long to diagnose. Even though it is rare, it is necessary to reconsider including HIV encephalopathy in the differential diagnosis of schizophrenia patients in routine clinical practice.

\section{REFERENCES}

[1] Hashimoto, R., Mukai, E., Yokomaku,-Y., Mamiya, N. and Hamaguchi, M. (2008) Clinical features and courses of 5 cases with HIV encephalopathy. Clinical Neurology, 48, 173-178.

[2] World Health Organization. (2008) World health statistics, 13-14.

[3] Nakashima, Y., Inoue, M. and Isse, K. (2002) Psychiatric problems of AIDS and related disorder. Japanese Journal of Clinical Psychiatry, 31, 925.

[4] Kopnisky, K.L. and Bao, J. (2007) HIV Preclinical therapeutics research: Central nervous system approaches. Journal of Neuroimmune Pharmacology, 2, 1.

[5] Bhaskaran, K., Mussini, C., Antinori, A., Walker, A.S., Dorrucci, M., Sabin, C., Phillips, A. and Porter, K. (2008) CASCADE collaboration. Change in the incidence and predictors of human immunodeficiency virus-associated dementia in the era of highly active antiretrovairal therapy. Annals of Neurology, 63, 213-221. doi:10.1002/ana.21225

[6] Uehira, T. and Shirasaka, T. (2001) HIV-associated cognitive-motor complex/AIDS dementia complex. Clinic All-Round, 50, 2738.

[7] Simpson, D.M. (1999) Human immunodeficiency virus-associated dementia: Review of pathogenesis, prophylaxis, and treatment studies of zidovudine therapy. Clinical Infectious Disease, 29, 19-34. doi:10.1086/520150

[8] Bottiggi, K.A., Chang, J.J., Schmitt, F.A., Avison, M.J., Mootoor, Y., Nath, A. and Berger J.R. (2007) The HIV dementia scale: Predictive power in mild dementia and HAART. Journal of the Neurological Sciences, 260, 1115. doi:10.1016/j.jns.2006.03.023 
[9] Bandaru, V.V., McArthur, J.C., Sacktor, N., Cutler, R.G., Knapp, E.L., Mattson, M.P. and Haughey, N.J. (2007) Associative and predictive biomarkers of dementia in
HIV-1-infected patients. Neurology, 68, 1481-1487. doi:10.1212/01.wnl.0000260610.79853.47 